TU-913

\title{
Partially Composite Higgs in Supersymmetry
}

\author{
Ryuichiro Kitano ${ }^{1}$, Markus A. Luty ${ }^{2,3}$, Yuichiro Nakai ${ }^{1}$ \\ ${ }^{1}$ Department of Physics, Tohoku University \\ Sendai Japan \\ ${ }^{2}$ Institut für Theoretische Physik der Universität Heidelberg \\ Hiedelberg, Germany \\ ${ }^{3}$ Physics Department, University of California Davis \\ Davis, California USA
}

\begin{abstract}
We propose a framework for natural breaking of electroweak symmetry in supersymmetric models, where elementary Higgs fields are semiperturbatively coupled to a strong superconformal sector. The Higgs VEVs break conformal symmetry in the strong sector at the $\mathrm{TeV}$ scale, and the strong sector in turn gives important contributions to the Higgs potential, giving rise to a kind of Higgs bootstrap. A Higgs with mass $125 \mathrm{GeV}$ can be accommodated without any fine tuning. A Higgsino mass of order the Higgs mass is also dynamically generated in these models. The masses in the strong sector generically violate custodial symmetry, and a good precision electroweak fit requires tuning of order $\sim 10 \%$. The strong sector has an approximately supersymmetric spectrum of hadrons at the $\mathrm{TeV}$ scale that can be observed by looking for a peak in the $W Z$ invariant mass distribution, as well as final states containing multiple $W, Z$, and Higgs bosons. The models also generically predict large corrections (either enhancement or suppression) to the $h \rightarrow \gamma \gamma$ width.
\end{abstract}




\section{Introduction}

Supersymmetry (SUSY) remains a compelling framework for addressing the naturalness problem of electroweak symmetry breaking [1]. The recent evidence for a $125 \mathrm{GeV}$ Higgs boson at the LHC [2] motivates us to ask whether such a Higgs mass is compatible with naturalness in the context of supersymmetry.

A $125 \mathrm{GeV}$ Higgs requires significant tuning in the minimal supersymmetric standard model (MSSM). The reason is that we need a significant radiative correction to the Higgs quartic from top and stops, the particles most strongly coupled to the Higgs:

$$
\Delta \lambda_{H} \sim \frac{y_{t}^{4} N_{c}}{16 \pi^{2}} \ln \frac{m_{\tilde{t}}}{m_{t}},
$$

where $N_{c}=3$. The stop mass is generally lighter than the other squark masses due to renormalization group effects, so this tends to push much or all of the superpartner spectrum is out of reach of the LHC. However, there is a good reason to think that this is not how nature works: such models are highly fine-tuned. The reason is that the large stop mass also generates a large quadratic term in the Higgs potential that must be tuned away:

$$
\Delta m_{H}^{2} \sim \frac{y_{t}^{2} N_{c}}{16 \pi^{2}} m_{\tilde{t}}^{2} \ln \frac{M^{2}}{m_{\tilde{t}}^{2}},
$$

where $M$ is a UV mass scale where the stop mass is generated. We note that such quadratic dependence of the Higgs mass parameter on large mass scales is precisely the naturalness problem that SUSY is supposed to address. Numerically, the tuning is of order a percent, even if the logarithm in Eq. (1.2) is not large. It is therefore well-motivated to consider possible mechanisms to reduce this tuning and study their experimental implications.

In order to generate a large quartic without fine-tuning, what is required are Higgs interactions that are stronger than typical perturbative interactions. We can see this from Eqs. (1.1) and (1.2): if we could increase $y_{t}$, then $\Delta \lambda_{H}$ increases as $y_{t}^{4}$ while $\Delta m_{H}^{2}$ only increases as $y_{t}^{2}$. In this paper, we consider the possibility that the Higgs is coupled to a strong sector, so the light Higgs is partially composite. This arises naturally in a supersymmetric model if the Higgs is coupled to a strong superconformal sector via operators

$$
W=\lambda_{u} H_{u} \mathcal{O}_{d}+\lambda_{d} H_{d} \mathcal{O}_{u},
$$

where $\mathcal{O}_{u, d}$ are operators in the conformal sector with the electroweak quantum numbers of $H_{u, d}$. The dimension $d$ of the operators $\mathcal{O}_{u, d}$ can be smaller than 2, so that 
the couplings above are relevant. This means that there is no UV problem with the interactions in Eq. (1.3) being stronger than perturbative interactions at the $\mathrm{TeV}$ scale. This theory can therefore easily accommodate heavy Higgs masses without fine tuning or UV problems. This is the primary motivation for considering such models.

On the other hand, the fact that these couplings are relevant introduces a new coincidence problem similar to the $\mu$-problem of the MSSM, namely why the scale of the SUSY-preserving terms Eq. (1.3) is near the weak scale. We will discuss a generalization of the Giudice-Masiero mechanism that can address this problem.

Ref. [3] studied the phenomenology of such models in the case where SUSY breaking at the $\mathrm{TeV}$ scale triggers confinement and dynamical electroweak symmetry breaking in the strong sector, which in turn induces VEVs for the elementary Higgs fields. (Such models were called "superconformal technicolor.") In this paper, we consider a different limit where the strong sector does not break electroweak symmetry in the limit $\lambda_{u, d} \rightarrow 0$. We assume that the dominant contribution to conformal symmetry breaking in the strong sector comes from the Higgs VEVs. The strong sector then gives important contributions to the effective potential of the elementary Higgs, so the model is a kind of Higgs bootstrap. These models have a very different phenomenology from superconformal technicolor models, as we will see below. Holographic 5D models with Higgs couplings of form Eq. (1.3) were studied in Ref. [4], assuming that SUSY is broken in the strong sector near the TeV scale. Models similar to ours were studied in Ref. [5] with particular attention to the case where the operators $\mathcal{O}_{u, d}$ have dimension near 2. See also Ref. [6] for a semi-perturbative model which appears as a dual description of a strongly coupled theory. Ref. [7], which appeared while this paper was being completed, also considers semi-perturbative conformal sectors, and is closely related to the present work.

In the presence of the interactions Eq. (1.3), electroweak symmetry breaking in the weakly-coupled sector induces breaking of conformal and electroweak symmetry in the strong sector at the $\mathrm{TeV}$ scale, in addition to explicit conformal symmetry breaking by soft SUSY breaking. We also consider the next-to-minimal supersymmetric standard model (NMSSM), where the VEV of the singlet is an important contribution to conformal symmetry breaking in the strong sector. These models eliminate a potential problem in superconformal technicolor, namely the presence of unstable potential in the strong sector [3]. In the absence of any tuning, we will see that the electroweak symmetry breaking masses in the strong sector are of the same size as the electroweak preserving masses, and the precision electroweak corrections are quite large. However, we will show that $\sim 10 \%$ tuning is sufficient to reduce the precision electroweak corrections, so the model is much less tuned than the MSSM. 
This paper is organized as follows. In $\S 2$, we describe specific models to set the stage for the more general discussion that follows. We consider the case where the dominant breaking of conformal symmetry in the strong sector comes from electroweak doublet or singlet Higgs fields. In $\S 3$, we give a general discussion of this class of models and estimate the corrections to the effective potential for the elementary Higgs fields. In $\S 4$, we give estimates for the precision electroweak corrections. In $\S 5$, we consider the phenomenology, and $\S 6$ gives our conclusions. In appendix, we discuss the contribution of soft SUSY breaking terms in the strong sector to the Higgs potential.

\section{Models}

We begin by presenting some specific models that illustrate the general ideas.

\subsection{Models with Custodial Symmetry}

The minimal model is based on an $S U(2)$ strong gauge group with 4 flavors (8 fundamentals). This theory is in the middle of the conformal window, and has no known weakly coupled description. The lowest-dimension chiral primary field (a meson) has dimension $\frac{3}{2}$. The fact that this is not close to the free-field dimension 1 is an indication that this is a truly strongly-coupled theory.

The gauge group of the model is

$$
S U(2)_{S} \times S U(2)_{L} \times S U(2)_{R} \times U(1)_{B-L}
$$

where the electroweak gauge group is embedded into $S U(2)_{L} \times S U(2)_{R} \times U(1)_{B-L}$ in the standard way, i.e. $S U(2)_{L}=S U(2)_{W}$ and $Y=T_{3 R}+B-L$. The MSSM Higgs fields $H_{u, d}$ are therefore contained in the field

$$
H \sim(1,2,2)_{0}
$$

This embedding of electroweak gauge group allows a natural custodial symmetry to act on the fields of the strong sector, namely the diagonal subgroup of $S U(2)_{L} \times$ $S U(2)_{R}$. On the other hand, multiplets of $S U(2)_{L} \times S U(2)_{R}$ are not complete GUT multiplets, so automatic gauge coupling constant unification is lost in these models.

There are various possibilities for the fields. One simple possibility is

$$
\begin{aligned}
& \Psi_{i} \sim(2,2,1)_{b}, \\
& \tilde{\Psi}_{i} \sim(2,1,2)_{-b},
\end{aligned}
$$


where $i=1,2$ and $b$ is the $B-L$ charge. In the custodial symmetry limit, we can write the Higgs coupling to this sector as

$$
W=\lambda_{i j} H \Psi_{i} \tilde{\Psi}_{j}
$$

The "meson" fields $\Psi \tilde{\Psi}$ have scaling dimension $\frac{3}{2}$, so the couplings $\lambda$ have dimension $+\frac{1}{2}$, i.e. they are relevant.

The Higgs VEVs break the conformal symmetry in the strong sector, giving positive supersymmetric masses to all matter fields in the strong sector. The $S U(2)_{S}$ gauge fields (and gauginos) are massless and confine, generating a dynamical superpotential

$$
W_{\mathrm{dyn}} \sim \lambda^{2} H_{u} H_{d}
$$

The model therefore generates a $\mu$ term dynamically.

There are also contributions to the Higgs potential arising from SUSY breaking scalar and gaugino masses in the strong sector. However, if SUSY breaking is transmitted to the strong sector at a high scale, large anomalous dimensions suppress the gaugino mass and universal scalar masses $[8,9]$. The result is that the soft masses at low energies must satisfy

$$
\sum_{i}\left(m_{i}^{2}+\tilde{m}_{i}^{2}\right)=0
$$

where $m_{i}^{2}\left(\tilde{m}_{i}^{2}\right)$ are the scalar masses for field $\Psi_{i}\left(\tilde{\Psi}_{i}\right)$. Such mass terms generally destabilize the vacuum of the strong sector [3], so we will assume that the strong sector masses generated by the Higgs VEVs dominate. These always give positive scalar masses, and there is no stability problem in these theories.

Even though SUSY breaking is subleading in the strong sector it can give important contributions to the Higgs potential, which in turn determines the scale $\Lambda$ of the strong sector. This is therefore a kind of "Higgs bootstrap."

To see whether this model is realistic, we need to estimate the size of these effects. Because the fixed point gauge coupling is strong at all scales, we expect that there is no hierarchy between the confinement scale $\Lambda$ and the scale of the masses induced by the VEVs. We can therefore estimate the terms in the effective Lagrangian using naïve dimensional analysis (NDA) [10]. ${ }^{1}$ This will be done in $\S 3$ below, after we have discussed several additional models to illustrate the range of possibilities. We will see that the contributions to the Higgs potential can be large enough to get a Higgs mass

\footnotetext{
${ }^{1}$ Exact results for $\mathcal{N}=2$ theories suggest that NDA is accurate in SUSY theories [9].
} 
of $125 \mathrm{GeV}$ without tuning, but the model requires tuning of order $10 \%$ to satsify precision electroweak constraints. This situation is more general than this specific model, and we will present a general analysis in $\S 4$ below.

A variation of this model is to allow electroweak preserving masses for the strong sector. These can be generated by adding a singlet Higgs field $S$ to the theory and writing the new superpotential terms

$$
\Delta W \sim S \Psi \Psi+S \tilde{\Psi} \tilde{\Psi}
$$

This is allowed only if we choose the $B-L$ charge $b$ to vanish. We can have electroweak preserving masses without introducing a singlet by adding " $\mu$ terms" to the superpotential, i.e. $\Delta W \sim \Psi \Psi+\tilde{\Psi} \tilde{\Psi}$. However, such terms have a different dimension from the Higgs couplings Eq. (2.4), and therefore have no reason to be of the same order.

The importance of adding electroweak-preserving masses is that the corrections to precision electroweak observables from the strong sector can be reduced if the electroweak-preserving masses are larger than the electroweak-breaking ones. We will see that this requires parametrically larger tuning than tuning toward a custodial symmetry limit, but it may work. Again, we will give a general analysis after we have presented several models.

Yet another variation is to have some of the strong fields be electroweak singlets. These can get a mass from coupling to $S$. This can somewhat reduce the precision electroweak corrections, and also the corrections to $h \rightarrow \gamma \gamma$, which we discuss in $\S 5.1$ below.

\subsection{Models with Unification}

It is natural to consider models where the strong fields come in complete $S U(5)$ multiplets, so that the theory unifies. A simple example of such a model is based on a strong $S U(3)$ gauge group with 6 flavors. This is again in the middle of the conformal window, and is a strongly-coupled theory.

The gauge group is

$$
S U(3)_{S} \times S U(5)_{\mathrm{SM}},
$$

where the standard model gauge group is embedded in $S U(5)_{\mathrm{SM}}$ in the standard way. 
The matter fields transform as

$$
\begin{aligned}
& \Psi \sim(3,5), \\
& \tilde{\Psi} \sim(\overline{3}, \overline{5}), \\
& \Sigma \sim(3,1), \\
& \tilde{\Sigma} \sim(\overline{3}, 1) .
\end{aligned}
$$

The $\mathbf{5}$ and $\overline{\mathbf{5}}$ fields decompose into doublet and triplet fields

$$
\Psi=(D, T), \quad \tilde{\Psi}=(\tilde{D}, \tilde{T}),
$$

and we can write superpotential couplings between the strong fields and Higgs fields as

$$
W=\lambda_{u} H_{u} \tilde{D} \Sigma+\lambda_{d} H_{d} D \tilde{\Sigma}+\lambda_{\Sigma} S \tilde{\Sigma} \Sigma+\lambda_{D} S \tilde{D} D+\lambda_{T} S \tilde{T} T
$$

Note that we need a singlet field $S$ to give masses to all the strong fields that are naturally the same size as those generated by Higgs VEVs.

One difficulty with this model is that the electroweak breaking masses in the strong sector cannot preserve custodial symmetry. This is because the electrically neutral component of the doublets can get an electroweak-breaking mass with the $\Sigma$

and $\tilde{\Sigma}$ fields, but there is no partner for the charged component of the doublets. As we will see below, these models require parametrically more tuning to satisfy precision electroweak constraints, but may still be viable.

\section{Partially Composite Higgs Potential}

In this section we give a general discussion of the contributions to the Higgs potential from the strong sector. Our discussion is valid for a general strong sector, but it is helpful to have the examples of the previous section in mind to understand the discussion.

We write the superpotential coupling as

$$
W=\kappa_{u}^{2-d} H_{u} \mathcal{O}_{d}+\kappa_{d}^{2-d} H_{d} \mathcal{O}_{u}
$$

where $d$ is the dimension of $\mathcal{O}_{u, d}$, so that $\kappa$ has dimensions of mass. We must have $d>1$ by unitarity. We assume $d<2$, so that these couplings are relevant, and normalize the operators so that the theory gets strong at the scale $\kappa$. The case $d \simeq 2$ 
is special, since the couplings are nearly dimensionless [5]. We will comment on this case below, but will focus mainly on the case where $2-d$ is not a small parameter. The strength of the couplings is then measured by

$$
\epsilon_{u, d} \sim\left(\frac{\kappa_{u, d}}{\Lambda}\right)^{2-d}
$$

where $\Lambda$ is the scale of conformal symmetry breaking in the strong sector (see below). These interactions cause the Higgs fields $H_{u, d}$ to mix with the strong conformal sector, which can give important contributions to the Higgs potential as we will see below.

$\Lambda$ is determined by conformal symmetry breaking mass terms in the strong sector. (By mass terms, we mean relevant terms in the Lagrangian involving only strong sector fields.) We will focus on the possibility that the mass terms in the strong sector induced by the Higgs VEVs via the coupling Eq. (3.1) are the largest breaking of conformal symmetry and trigger the exit from the CFT fixed point. Because the Higgs VEVs preserve SUSY, the strong sector is approximately supersymmetric. The strong sector then in turn gives important contributions to the Higgs potential, this is a kind of Higgs bootstrap.

It is also possible to have conformal symmetry breaking that does not violate electroweak symmetry from explicit $\mu$-like terms, or from the VEV of a perturbative singlet field. There is an interesting class of models where this is the dominant source of conformal symmetry breaking in the strong sector. Again, the strong sector is naturally approximately supersymmetric in this case.

Finally, there is soft SUSY breaking in the strong sector. As discussed in $\S 2.1$, if this is the dominant source of conformal symmetry breaking in the strong sector, then there are difficulties with vacuum stability. These can be overcome with some additional structure [3] but we will focus on the case where the dominant source of conformal symmetry breaking is supersymmetric.

\subsection{Higgs Bootstrap}

We first consider the situation where electroweak breaking Higgs VEVs trigger the exit from the CFT. This occurs in the first model of $\S 2.1$ above, but may also occur in models with singlet fields if their VEVs are subdominant. In this case we have

$$
\epsilon \sim \frac{\Lambda}{4 \pi v}
$$

where $\epsilon \sim \epsilon_{u, d}$ and we assume $v_{u} \sim v_{d}$. We can understand this from the fact that in the double limit $\epsilon \sim 1$ and $4 \pi v \sim \Lambda$ both the relevant interactions and the Higgs mass contributions are strong at the scale $\Lambda$. 
Note that the Higgs VEV is a SUSY-preserving mass in the strong sector. We therefore first consider the dynamics of the strong sector in the SUSY limit. The scale of strong dynamics is determined by the Higgs VEV, and can be parameterized by a holomorphic superfield $\Lambda$. These models will generally generate a dynamical superpotential. We expect a dynamical superpotential to be generated if there is a holomorphic candidate that is invariant under all symmetries, in particular the conformal $U(1)_{R}$. Note that we are assuming that the Higgs couplings give mass to all matter fields in the strong sector. This means that there is a holomorphic mass scale $\Lambda(H)$ for the theory that depends on the Higgs fields. For holomorphic quantities, $U(1)_{R}$ invariance is equivalent to dimensional analysis, so there is always an allowed dynamical superpotential of the form

$$
\Delta W_{\mathrm{dyn}} \sim \frac{\Lambda^{3}(H)}{16 \pi^{2}}
$$

The most general form of the dynamical scale compatible with all symmetries is

$$
\Lambda^{6-2 d}(H) \sim 16 \pi^{2} \kappa_{u}^{2-d} \kappa_{d}^{2-d} H_{u} H_{d} .
$$

In the explicit models of $\S 2.1$ we can verify that the usual dynamical superpotential is indeed generated and has this form. The factors of $4 \pi$ in Eqs. (3.4) and (3.5) follow from NDA. We can understand them by noting that the interactions Eq. (3.1) are strong at the scale $\Lambda$ in the limit $\epsilon_{u, d} \rightarrow 1,4 \pi v \rightarrow \Lambda$.

If we replace the Higgs fields by their VEVs, we get a simpler and more intuitive expression in terms of $\epsilon_{u, d}$ :

$$
\Lambda \sim 4 \pi\left(\epsilon_{u} \epsilon_{d}\right)^{1 / 2}\left(v_{u} v_{d}\right)^{1 / 2} \sim 4 \pi \epsilon v
$$

which is consistent with Eq. (3.3). However, Eq. (3.5) must be used to get the correct form of the $H_{u, d}$ dependence.

The superpotential Eq. (3.4) is non-analytic for at $H_{u, d}=0$, corresponding to the fact that it is obtained by integrating out particles that get a mass from the Higgs VEVs. An interesting special case is $d=\frac{3}{2}$, for which we have

$$
W_{\text {dyn }}\left(d=\frac{3}{2}\right) \sim \kappa_{u}^{1 / 2} \kappa_{d}^{1 / 2} H_{u} H_{d}
$$

i.e. the superpotential is a pure $\mu$-term. Note that this is what happens in the first model of $\S 2.1$ above. For general $d$ the superpotential Eq. (3.4) generates a Higgsino mass of order

$$
\mu_{\text {dyn }} \sim \frac{\partial^{2} W}{\partial H^{2}} \sim \frac{\Lambda^{3}}{(4 \pi v)^{2}} \sim \epsilon^{2} \Lambda
$$


We will see below that this gives a Higgsino mass term of order the Higgs mass, giving a viable solution to the $\mu$-problem. This is certainly an attractive feature of this class of models.

The supersymmetric contribution to the Higgs potential from the dynamical superpotential has the form

$$
V_{\mathrm{dyn}} \sim \frac{|\Lambda(H)|^{6}}{(4 \pi)^{4}} \frac{H_{u}^{\dagger} H_{u}+H_{d}^{\dagger} H_{d}}{\left|H_{u} H_{d}\right|^{2}} \sim H^{2 d /(3-d)} .
$$

The potential is positive-definite, and for $d>\frac{3}{2}$ the potential grows faster than $H^{2}$ so we can obtain a stable electroweak breaking minimum by adding negative Higgs quadratic terms. We will be considering several different contributions to the Higgs potential that can balance each other in various combinations. A good way to understand the relative sizes of the various contributions is to look at the second derivative of the potential. All of the contributions to the potential we will study go as a power of the Higgs fields. The minimization is in general dominated by balancing two different power-law contributions to the potential, so the second derivative of each of them at the minimum will be the same up to factors of order 1. This is also equal to the physical Higgs mass (again up to factors of order 1), so this allows us to estimate the physical parameters associated with a given contribution to the potential, assuming it is important for determining the Higgs VEVs. In the present case, we have

$$
V_{\mathrm{dyn}}^{\prime \prime} \sim \frac{1}{(4 \pi)^{4}}\left(4 \pi \kappa^{2-d}\right)^{6 /(3-d)} v^{(4 d-6) /(3-d)} \sim \frac{\Lambda^{6}}{(4 \pi v)^{4}} \sim \epsilon^{4} \Lambda^{2},
$$

where we have used the relations Eqs. (3.5) and (3.6) in the last steps. If this contribution to the potential is important for stabilizing the Higgs mass, we have

$$
\epsilon \sim\left(\frac{m_{h}}{4 \pi v}\right)^{1 / 3}
$$

For numerical estimates, we use $4 \pi v \simeq 2 \mathrm{TeV}$, which is the value of the technirho mass in scaled-up QCD. For $m_{h} \simeq 125 \mathrm{GeV}$ we then obtain $\epsilon \sim 0.4$ and $\Lambda \sim 800 \mathrm{GeV}$. In any stabilization where this contribution is important, the Higgsino mass Eq. (3.8) is related to the Higgs mass by

$$
\frac{\mu_{\mathrm{dyn}}}{m_{h}} \sim 1
$$

In other words, the dynamical superpotential gives a perfect parametric solution to the $\mu$-problem. 
The interactions in Eq. (3.1) are rather close to becoming strong at the scale $\Lambda$. This coincidence problem will be addressed in $§ 3.3$. One may also worry about performing an expansion for small $\epsilon_{u, d}$ for such large values. However, observables are suppressed by large powers of this suppression, making the estimates more plausible. However, these estimates clearly have large uncertainties, and should be regarded as enlightened order-of-magnitude estimates.

We now consider the contributions to the Higgs potential from SUSY breaking in the strong sector. There can be $A$ terms associated with the interaction Eq. (3.1) of the form

$$
\Delta \mathcal{L}=\kappa_{u}^{2-d} A_{u} H_{u} \mathcal{O}_{d}+\kappa_{d}^{2-d} A_{d} H_{d} \mathcal{O}_{u}+\text { h.c. }
$$

These $A$ terms are not strongly renormalized by the strong sector, since they are proportional to a relevant coupling that is small in the UV. It is therefore natural to have $A_{u, d} \sim \mathrm{TeV}$. The $A$ terms give a contribution to the Higgs potential

$$
V_{A} \sim \frac{\Lambda^{3}(H)}{16 \pi^{2}}\left(A_{u}+A_{d}\right)+\text { h.c. } \sim H^{3 /(3-d)} .
$$

Potential terms that are real parts of holomorphic functions such as this always have unstable directions. For $d<\frac{3}{2}$, Eq. (3.14) grows slower than $H^{2}$ and we can get a stable electroweak breaking minimum by balancing this against positive SUSYbreaking $H^{2}$ terms in the potential. We have

$$
V_{A}^{\prime \prime} \sim \frac{A}{(4 \pi)^{2}}\left(4 \pi \kappa^{2-d}\right)^{3 /(3-d)} v^{(2 d-3) /(3-d)} \sim \frac{A \Lambda^{3}}{(4 \pi v)^{2}} \sim \epsilon^{2} A \Lambda .
$$

If this contribution to the potential is important for stabilizing the Higgs mass, we have

$$
\epsilon \sim\left(\frac{\Lambda}{A}\right)^{1 / 4}\left(\frac{m_{h}}{4 \pi v}\right)^{1 / 2}
$$

Note that $A \lesssim \Lambda$, otherwise conformal symmetry breaking in the strong sector is dominated by the $A$ terms. The Higgsino mass Eq. (3.8) is now related to the Higgs mass as

$$
\frac{\mu_{\mathrm{dyn}}}{m_{h}} \sim \epsilon\left(\frac{\Lambda}{A}\right)^{1 / 2}
$$

The Higgsino mass is not parametrically of order the Higgs mass, but the values of $\epsilon$ are not very large, and we can easily get a viable model without an additional contribution to the Higgsino mass. 
Other types of SUSY breaking terms in the strong sector generally have large anomalous dimensions. An exception are scalar mass-squared terms proportional to flavor generators (not including $U(1)_{R}$ ), which are not renormalized. If we assume that SUSY breaking originates at scales far above the $\mathrm{TeV}$ scale, these are the only SUSY breaking terms in the strong sector that are naturally of order the TeV scale. (Other soft mass terms are generally suppressed at an IR attractive fixed point, so this is a natural scenario.)

The non-renormalization of scalar mass-squared terms proportional to flavor generators can be understood from the fact that these mass terms can be written as the $D$-term for a flavor gauge superfield. Under conformal transformations, the flavor gauge fields have dimension 0, so the soft mass-squared term have dimension 2 . Similarly, the combination $\kappa^{2-d} H$ is a chiral primary field of dimension $3-d$. This constraints how these fields can appear in the effective theory below the scale $\Lambda$. (This is discussed in detail in the Appendix.) The resulting potential can be expanded in powers of the soft masses if these are a subleading contribution to conformal symmetry breaking in the strong sector, and we get

$$
V_{\mathrm{soft}} \sim \frac{1}{16 \pi^{2}} m_{\mathrm{soft}}^{2}\left(4 \pi \kappa^{2-d} H\right)^{2 /(3-d)} .
$$

This contribution to the potential is not directly expressible in terms of the holomorphic scale $\Lambda(H)$, and the functional form is not calculable (see Appendix).

In the models that we construct, the symmetries do not prevent a nontrivial potential of this form. We can choose this potential to stabilize or destabilize $H=0$ by choosing the sign of $m_{\mathrm{soft}}^{2}$. Because we are assuming that the soft mass-squared terms are a subleading contribution to conformal symmetry breaking in the strong sector, negative mass-squared terms will not induce a vacuum instability in the strong sector. Eq. (3.18) grows more slowly than $H^{2}$ for $d<2$, so we can obtain a stable electroweak breaking minimum by $m_{\text {soft }}^{2}<0$ so that this contribution destabilizes $H=0$ and balancing it against a positive quadratic term for $H$. The Higgs mass scale is then

$$
V_{\mathrm{soft}}^{\prime \prime} \sim m_{\mathrm{soft}}^{2} \frac{\Lambda^{2}}{(4 \pi v)^{2}} \sim \epsilon^{2} m_{\mathrm{soft}}^{2} .
$$

To summarize, the contributions to the Higgs potential from the strong sector are given by $V_{\text {dyn }}, V_{A}, V_{\text {soft }}$. In the above we briefly discussed balancing each of these with tree-level Higgs mass terms

$$
V_{\text {tree }}=m_{H u}^{2}\left|H_{u}\right|^{2}+m_{H d}^{2}\left|H_{d}\right|^{2}+B \mu\left(H_{u} H_{d}+\text { h.c. }\right) \sim m_{H}^{2} H^{2} .
$$


However, other combinations are possible, and we will summarize all possibilities. We neglect the Higgs quartic terms from the standard model gauge interactions, since these are far too small to give $m_{h} \simeq 125 \mathrm{GeV}$.

- $V_{\text {dyn }}$ and $V_{\text {tree }}$ : This can work for $\frac{3}{2}<d<2$. We obtain

$$
\epsilon \sim\left(\frac{m_{h}}{4 \pi v}\right)^{1 / 3} \sim 0.4
$$

which gives $\Lambda \sim 800 \mathrm{GeV}$ for $m_{h} \simeq 125 \mathrm{GeV}$. The dynamically generated Higgsino mass is related to the Higgs mass by

$$
\frac{\mu_{\mathrm{dyn}}}{m_{h}} \sim 1
$$

so this gives a perfect parametric solution to the $\mu$-problem.

- $V_{A}$ and $V_{\text {tree }}$ : This can work for $1<d<\frac{3}{2}$. We obtain

$$
\epsilon \sim\left(\frac{m_{h}}{4 \pi v}\right)^{1 / 2}\left(\frac{A}{\Lambda}\right)^{-1 / 4} \sim 0.25\left(\frac{A}{\Lambda}\right)^{-1 / 4}
$$

which gives $\Lambda \sim 500 \mathrm{GeV} \times(A / \Lambda)^{-1 / 4}$. We must have $A / \Lambda \lesssim 1$, since $A \sim \Lambda$ corresponds to conformal symmetry breaking dominated by $A$. Consistency requires that $V_{\text {dyn }}$ is subdominant, which occurs for

$$
\frac{A}{\Lambda} \gtrsim\left(\frac{m_{h}}{4 \pi v}\right)^{2 / 3} \sim 0.16
$$

The dynamically generated $\mu$-term is given by

$$
\frac{\mu_{\mathrm{dyn}}}{m_{h}} \sim\left(\frac{m_{h}}{4 \pi v}\right)^{1 / 2}\left(\frac{A}{\Lambda}\right)^{-3 / 4} \lesssim 1
$$

where the bound follows from Eq. (3.24). The dynamically generated $\mu$-term is therefore parametrically too small in this limit. However, the suppression factors are not large, and it is possible that the numerical value is sufficiently large.

- $V_{\text {soft }}$ and $V_{\text {tree }}$ : This can work for any $1<d<2$. We obtain

$$
\epsilon \sim\left(\frac{m_{h}}{4 \pi v}\right)^{1 / 2}\left(\frac{m_{\mathrm{soft}}}{\Lambda}\right)^{-1 / 2} \sim 0.25\left(\frac{m_{\mathrm{soft}}}{\Lambda}\right)^{-1 / 2}
$$


We must have $m_{\text {soft }} / \Lambda \lesssim 1$, since $m_{\text {soft }} \sim \Lambda$ corresponds to conformal symmetry breaking dominated by $m_{\text {soft }}$. Consistency requires that $V_{\text {dyn }}$ is subdominant, which occurs for

$$
\frac{m_{\text {soft }}}{\Lambda} \gtrsim\left(\frac{m_{h}}{4 \pi v}\right)^{1 / 3} \sim 0.4 .
$$

The dynamically generated $\mu$-term is given by

$$
\frac{\mu_{\mathrm{dyn}}}{m_{h}} \sim\left(\frac{m_{h}}{4 \pi v}\right)^{1 / 2}\left(\frac{m_{\mathrm{soft}}}{\Lambda}\right)^{-3 / 2} \lesssim 1,
$$

where the bound follows from Eq. (3.27).

- $V_{\text {dyn }}$ and $V_{A}$ : This can work for $\frac{3}{2}<d<2$. We obtain

$$
\epsilon \sim\left(\frac{A}{\Lambda}\right)^{1 / 2} \sim\left(\frac{m_{h}}{4 \pi v}\right)^{1 / 3} \sim 0.4 .
$$

Because $V_{\mathrm{dyn}}$ is part of the stabilization of the potential, we have $\mu_{\mathrm{dyn}} \sim m_{h}$.

- $V_{\text {dyn }}$ and $V_{\text {soft }}$ : This can work for any $1<d<2$. We choose $m_{\text {soft }}^{2}<0$ to destabilize $H=0$, and $V_{\text {dyn }}$ provides the stabilizing potential. We obtain

$$
\epsilon \sim \frac{m_{\text {soft }}}{\Lambda} \sim\left(\frac{m_{h}}{4 \pi v}\right)^{1 / 3} \sim 0.4 .
$$

We also have $\mu_{\mathrm{dyn}} \sim m_{h}$.

- $V_{A}$ and $V_{\text {soft }}$ : This combination is not expected to work because $V_{A}$ is always destabilizing and grows as a larger power of $H$ than $V_{\text {soft }}$.

The spectrum of the strong sector is approximately supersymmetric in all of these cases, even the ones in which the Higgs VEVs are determined by SUSY breaking interactions. The reason is that we are always choosing parameters so that the Higgs VEVs are the dominant source of conformal breaking in the strong sector. This is motivated by the fact that supersymmetric masses in the strong sector naturally give a stable vacuum for the strong sector. If SUSY breaking dominates the breaking of conformal invariance in the strong sector, this stability is lost in the simplest models (recall the discussion in $\S 2.1$ ). 


\subsection{Electroweak Preserving Masses}

We now consider the case where there is an electroweak-preserving contribution to the mass scale in the strong sector that is the dominant source of conformal symmetry breaking. This can arise naturally from the VEV of a singlet Higgs field $S$ coupled to the CFT via a superpotential interaction similar to Eq. (3.1):

$$
\Delta W=\kappa_{S}^{2-d} S \mathcal{O}_{S}
$$

where $\mathcal{O}_{S}$ is a CFT operator with the same dimension $d$ as $\mathcal{O}_{u, d}$. Soft SUSY breaking naturally generates a $\mathrm{VEV}$ for $S$ that can be somewhat larger than $v$. It is also possible to have a $\mu$-like term $\Delta W \sim \mathcal{O}_{S}$, but in general this would not be expected

to give mass terms with the same order of magnitude as the contribution from the Higgs VEVs from Eq. (3.1).

In the limit where we neglect $\left\langle H_{u, d}\right\rangle$ the strong scale is given by

$$
\Lambda_{0}^{3-d} \sim 4 \pi \kappa_{S}^{2-d}\langle S\rangle
$$

This is an arbitrary parameter of the theory.

The dominant source of conformal symmetry breaking in the strong sector is assumed to come from the singlet VEV, which is supersymmetric. We therefore begin by analyzing the strong sector in the SUSY limit. The holomorphic strong scale is

$$
\Lambda(H)=\Lambda_{0}\left[1+c_{1} \epsilon_{u} \epsilon_{d} \frac{16 \pi^{2} H_{u} H_{d}}{\Lambda_{0}^{2}}+c_{2}\left(\epsilon_{u} \epsilon_{d} \frac{16 \pi^{2} H_{u} H_{d}}{\Lambda_{0}^{2}}\right)^{2}+\cdots\right]
$$

where $c_{1,2}$ are order-1 numbers calculable in specific models. The condition that the Higgs contribution to the dynamical scale is subleading is then

$$
\delta_{H} \sim\left(\epsilon \frac{4 \pi v}{\Lambda_{0}}\right)^{2} \lesssim 1
$$

This is the (square of) the expansion parameter in Eq. (3.33). Note that having $\delta_{H}<1$ always requires some parametric tuning, since minimizing the potential with $c_{1,2} \sim 1$ gives $\delta_{H} \sim 1$. To get $\delta_{H} \ll 1$ we need a smaller quadratic term, which requires unnatural cancellations. In fact, $\delta_{H}$ is precisely the measure of fine tuning in these models. Just as in composite Higgs models, we are accepting a mild tuning as the price for a model that has a light Higgs particle and good precision electroweak fit. 
We again have a dynamical superpotential of the form Eq. (3.4). The corresponding supersymmetric contribution to the Higgs potential is

$$
\begin{aligned}
V_{\mathrm{dyn}}= & \left|\frac{1}{16 \pi^{2}} \frac{\partial \Lambda^{3}(H)}{\partial H}\right|^{2} \\
\sim & \left(\epsilon_{u} \epsilon_{d}\right)^{2}\left[\Lambda_{0}^{2}\left(H_{u}^{\dagger} H_{u}+H_{d}^{\dagger} H_{d}\right)\right. \\
& \left.\quad+16 \pi^{2} \epsilon_{u} \epsilon_{d}\left(H_{u}^{\dagger} H_{u}+H_{d}^{\dagger} H_{d}\right)\left(H_{u} H_{d}+\text { h.c. }\right)+\cdots\right] .
\end{aligned}
$$

Note that we know the functional form of the potential because we know the functional form of the holomorphic scale $\Lambda(H)$. There are corrections to the $H$ Kähler potential, but they are smaller than the tree-level $H$ kinetic term.

We would like to consider the possibility that Eq. (3.35) gives the leading contribution to the Higgs quartic. This scenario always requires some tuning, because it requires the $H^{2}$ terms to be somewhat smaller than in Eq. (3.35) so that the Higgs VEV is a subleading contribution to $V_{\mathrm{dyn}}$. However, the model can work with only very mild tuning. If this potential is part of the stabilization of the Higgs VEVs, the Higgs mass parameter is

$$
V_{\mathrm{dyn}}^{\prime \prime} \sim\left(\epsilon_{u} \epsilon_{d}\right)^{3}(4 \pi v)^{2}
$$

For a Higgs mass of $125 \mathrm{GeV}$ this gives $\left(\epsilon_{u} \epsilon_{d}\right)^{1 / 2} \sim 0.4$ as above. The measure of tuning is precisely the expansion parameter $\delta_{H}$ in Eq. (3.34). There is a trade-off between naturalness and predictability, but we get a plausible scenario for moderately small values of $\delta_{H}$, e.g. $\delta_{H} \sim 0.2$.

The quartic Higgs interaction in Eq. (3.35) is not positive definite. The full dynamically generated potential is positive definite, so the question is whether there is a stable electroweak-breaking minimum with VEVs sufficiently small that the quartic term dominates. It is easy to see there is such a local minimum even in the limit where the SUSY quartic vanishes, for a sufficiently large $B \mu$ term of the correct sign. Specifically, for the potential

$$
\begin{gathered}
V=m_{H u}^{2} H_{u}^{\dagger} H_{u}+m_{H d}^{2} H_{d}^{\dagger} H_{d}-B \mu\left(H_{u} H_{d}+\text { h.c. }\right) \\
+\lambda\left(H_{u}^{\dagger} H_{u}+H_{d}^{\dagger} H_{d}\right)\left(H_{u} H_{d}+\text { h.c. }\right)
\end{gathered}
$$

we find a local stable electroweak breaking minimum for $H_{u} H_{d}>0$ provided that

$$
B \mu>\frac{5 \lambda v^{2}}{8}\left[1+\frac{1}{5} \cos (4 \beta)\right] .
$$


This solution is in general only a local minimum, since there is an unstable $D$-flat direction $\left|H_{u}\right|=\left|H_{d}\right|, H_{u} H_{d}<0$. The global minimum can be far away in field space, so at least some of these solutions are expected to be cosmologically acceptable. We will not explore this issue further here.

The dynamical superpotential also generates a Higgsino mass

$$
\mu_{\mathrm{dyn}} \sim \epsilon_{u} \epsilon_{d} \Lambda_{0}
$$

Eq. (3.36) then implies that

$$
\frac{\mu_{\mathrm{dyn}}}{m_{h}} \sim \frac{1}{\delta_{H}^{1 / 2}}
$$

The dynamically generated $\mu$-term is somewhat larger than the Higgs mass, which still gives a good solution to the $\mu$-problem.

We now turn to SUSY breaking contributions to the potential. Because we are assuming that the Higgs VEVs are a subleading contribution to the potential, the potential can always be expanded in terms of gauge-invariant combinations of Higgs fields, so the dominant contributions will be qualitatively similar to $V_{\text {dyn }}$ discussed above. In particular, we always require a tuning of order $\delta_{H}$ given by Eq. (3.34) to make the Higgs contribution subleading.

Specifically, we concerned with $A$ terms of the form

$$
\Delta \mathcal{L}=\kappa_{S}^{2-d} A_{S} S \mathcal{O}_{S}+\text { h.c. }
$$

and scalar mass-squared terms $m_{\text {soft }}^{2}$ proportional to flavor generators. These give rise to a potential of the form

$$
V_{A, \text { soft }} \sim \xi_{A, \text { soft }} \frac{\Lambda_{0}^{4}}{16 \pi^{2}}\left[1+\left(\frac{4 \pi \epsilon H}{\Lambda_{0}}\right)^{2}+\left(\frac{4 \pi \epsilon H}{\Lambda_{0}}\right)^{4}+\cdots\right] .
$$

We assume that the SUSY breaking terms are a subleading contribution to conformal symmetry breaking in the strong sector, so we can expand the suppression factor in powers of the SUSY breaking masses:

$$
\begin{aligned}
\xi_{A} & \sim \frac{A_{S}}{\Lambda_{0}} \\
\xi_{\text {soft }} & \sim \frac{m_{\text {soft }}^{2}}{\Lambda_{0}^{2}} .
\end{aligned}
$$

$V_{A}$ is the real part of a holomorphic function, and therefore cannot stabilize the Higgs VEV. (The $H^{2}$ terms in $V_{A}$ are proportional to $H_{u} H_{d}+$ h.c. and the quartic terms 
are proportional to $\left(H_{u} H_{d}\right)^{2}+$ h.c.) As with $V_{\text {dyn }}$, we need parametric tuning of order $\delta_{H}$ given by Eq. (3.34) to make the Higgs VEV contribution subleading.

We will assume that either $V_{\text {dyn }}$ or $V_{\text {soft }}$ dominates the Higgs quartic. We then have

$$
m_{h}^{2} \sim \lambda v^{2} \sim 16 \pi^{2} \xi_{\text {dyn }, \text { soft }} \epsilon^{4} v^{2}
$$

where $\xi_{\text {soft }}$ is given in Eq. (3.44) and

$$
\xi_{\text {dyn }} \sim \epsilon^{2}
$$

The two possible origins for the Higgs quartic are then as follows.

- $V_{\text {dyn }}$ : This requires

$$
\epsilon \sim\left(\frac{m_{h}}{4 \pi v}\right)^{1 / 3} \sim 0.4
$$

The scale $\Lambda_{0}$ depends on the degree of fine-tuning:

$$
\Lambda_{0} \sim \delta_{H}^{-1 / 2} \epsilon 4 \pi v \sim 800 \mathrm{GeV} \times \delta_{H}^{-1 / 2}
$$

- $V_{\text {soft }}$ : This requires

$$
\epsilon \sim\left(\frac{m_{h}}{4 \pi v}\right)^{1 / 2}\left(\frac{m_{\text {soft }}}{\Lambda_{0}}\right)^{-1 / 2} \sim 0.25\left(\frac{m_{\text {soft }}}{\Lambda_{0}}\right)^{-1 / 2} .
$$

Requiring that the soft mass contribution dominates $V_{\text {dyn }}$, we obtain

$$
\frac{m_{\mathrm{soft}}}{\Lambda_{0}} \gtrsim\left(\frac{m_{h}}{4 \pi v}\right)^{1 / 3} \sim 0.4
$$

We then have

$$
\Lambda_{0} \sim 500 \mathrm{GeV} \times \delta_{H}^{-1 / 2}\left(\frac{m_{\mathrm{soft}}}{\Lambda_{0}}\right)^{-1 / 2} \lesssim 800 \mathrm{GeV} \times \delta_{H}^{-1 / 2} .
$$

\subsection{Coincidence Problem}

A potential problem with this framework is that $\kappa_{u, d}$ in Eq. (3.1) are dimensionful parameters that must be near the $\mathrm{TeV}$ scale in order to have a successful model. This is analogous to the $\mu$-problem in the MSSM, where a SUSY-preserving mass must be of order the SUSY breaking masses. We have seen that in our models a Higgsino mass 
of the correct size can be dynamically generated, so there is no need for a $\mu$-term, so we have traded one coincidence problem for another.

Here we point out that the mass scale $\kappa_{u, d}$ can naturally be near the TeV scale by a generalization of the Giudice-Masiero mechanism [3]. We focus on the minimal model discussed in $\S 2.1$ and let us consider a SUSY breaking field $X$ with a nonzero $F$-term $\left\langle F_{X}\right\rangle$ in the hidden sector. The visible sector SUSY breaking including $m_{\text {soft }}$ and $m_{H}$ is given by

$$
M_{\mathrm{SUSY}} \sim \frac{\left\langle F_{X}\right\rangle}{M_{\mathrm{med}}}
$$

where $M_{\text {med }}$ is the mediation scale. In addition, we assume that the hidden sector contains a field $Y$ with the following expectation values:

$$
\langle Y\rangle \sim\left\langle F_{X}\right\rangle^{1 / 2}
$$

and $\left\langle F_{Y}\right\rangle$ sufficiently small. Then the interaction Eq. (3.1) can be generated by the superpotential

$$
W_{\text {eff }} \sim \frac{1}{M_{\mathrm{med}}^{1 / 2}} Y H \Psi_{i} \tilde{\Psi}_{j},
$$

and we have $\kappa \sim M_{\mathrm{SUSY}}$. The correct size of $\langle Y\rangle$ can be realized with the hidden sector superpotential:

$$
W_{\mathrm{hid}}=c_{1} X+\frac{c_{2}}{M_{\mathrm{med}}} Y^{4}
$$

where $c_{1,2}$ are order-1 coupling constants.

Another possibility to solve the coincidence problem is to consider strong sectors where the operators $\mathcal{O}_{u, d}$ have dimension $d \simeq 2[5]$. This does not occur in stronglycoupled SUSY QCD models, but is perfectly allowed in more general strong theories. In such models, the couplings $\kappa_{u, d}$ are nearly dimensionless, requiring a milder coincidence. However, we have seen that we need this coupling to be rather strong at the strong scale $\Lambda: \epsilon \sim 0.4$, meaning that the coupling is about half its strong-coupling value. This means that in general the coupling is running fast at the scale $\Lambda$, and we still have a coincidence problem. Alternatively, we could assume that the couplings $\kappa_{u, d}$ are slightly relevant and approach a strong fixed point, giving a robust explanation for the large value of the coupling. At this fixed point the Higgs fields $H_{u, d}$ become conformal operators with dimension near 1 . This implies that they are nearly decoupled from the CFT, in contradiction with what is required for phenomenology. We conclude that it is not clear whether $d \simeq 2$ really gives a viable theory without a coincidence problem. 


\section{Precision Electroweak Tests}

We now consider the constraints on this scenario from precision electroweak tests. Our strong sector does not couple directly to the quarks and leptons, and therefore the only important corrections are the $S$ and $T$ parameters [11]. (In particular, the correction to $Z \rightarrow \bar{b} b$ is negligible.) These are simply effective operators that arise from integrating out the strong sector. We cannot perform a precise computation, but we can estimate them using NDA.

\subsection{T Parameter}

We now estimate the $T$ parameter, which gives the strongest constraint on this class of models.

We first consider models without electroweak-preserving masses in the strong sector, as discussed in $§ 3.1$. We assume that the strong sector has a custodial symmetry in the limit where the Higgs couplings are turned off, as in the models of $\S 2.1$. In general, the Higgs couplings do not preserve the custodial symmetry $\left(\kappa_{u} \neq \kappa_{d}\right)$, and it is not natural to have $v_{u}=v_{d}$. This means that custodial symmetry is generically broken in the strong sector. This leads to large corrections to the $T$ parameter, but these can be made sufficiently small with mild tuning. The strong sector will have custodial symmetry if accidentally $\kappa_{u}^{2-d} v_{u} \simeq \kappa_{d}^{2-d} v_{d}$. The tuning required is measured by

$$
\delta_{C}=\frac{1}{2} \frac{\kappa_{u}^{2-d} v_{u}-\kappa_{d}^{2-d} v_{d}}{\kappa_{u}^{2-d} v_{u}+\kappa_{d}^{2-d} v_{d}} .
$$

The contribution to the $T$ parameter from the strong sector is estimated by the contribution of $N$ doublets of fermions with electroweak breaking masses of order $\Lambda$, with custodial breaking mass splitting of order $\Delta m \sim \delta_{C} \Lambda$. This is similar in spirit to estimating the short-distance contribution to the $S$ parameter in technicolor models using the contribution from "constituent techniquarks," which is known to give an accurate answer both parametrically and numerically. In the present case, this gives

$$
\Delta T \simeq \frac{N(\Delta m)^{2}}{12 \pi s_{W}^{2} c_{W}^{2} m_{Z}^{2}} \sim 0.4\left(\frac{N}{4}\right)\left(\frac{\epsilon}{0.4}\right)^{2}\left(\frac{\delta_{C}}{0.1}\right)^{2} .
$$

We see that the corrections to the $T$ parameter are moderate at the price of roughly $10 \%$ tuning.

We next consider the case where the electroweak-preserving masses in the strong sector dominate. The important parameter in this case is $\delta_{H}$, the square of the ratio 
of the electroweak-preserving and electroweak breaking masses (see Eq. (3.34)). This ratio can only be made small by tuning, and $\delta_{H}$ is a measure of this tuning. In this case, we estimate the tuning by $N$ doublets of fermions with electroweak breaking mass $\Delta m \sim \delta_{H}^{1 / 2} \Lambda_{0}[12]:$

$$
\Delta T \simeq \frac{13}{480 \pi s_{W}^{2} c_{W}^{2} m_{Z}^{2}} \frac{N \Delta m^{4}}{\Lambda_{0}^{2}} \sim 1.2\left(\frac{N}{4}\right)\left(\frac{\epsilon}{0.4}\right)^{2}\left(\frac{\delta_{H}}{0.1}\right),
$$

where we have used

$$
\frac{(\Delta m)^{4}}{\Lambda_{0}^{2}} \sim \delta_{H}^{2} \Lambda_{0}^{2} \sim \delta_{H} \epsilon^{2}(4 \pi v)^{2} .
$$

Note that the reduction in $\Delta T$ is only linear in $\delta_{H}$, so it parametrically more tuning is required to get a sufficiently small $T$ parameter in this case. Of course, all of these estimates have order-1 uncertainties, but the tuning required to get a phenomenologically viable $T$ parameter is significantly less than the $1 \%$ (or less) tuning of the MSSM.

\section{2 $S$ Parameter}

We estimate the contribution to the $S$ parameter from $N$ doublets of fermions with electroweak breaking masses. We then obtain

$$
\Delta S \sim \frac{N}{6 \pi} \sim 0.2\left(\frac{N}{4}\right) .
$$

In the case where electroweak preserving masses dominate, the $S$ parameter is suppressed by a factor of $\delta_{H}$ compared to the above estimate.

We expect the strong sector contributions to both the $S$ and $T$ parameters to be positive. This is true for perturbative contribution from electroweak breaking multiplets, and also holds for holographic theories where $S$ and $T$ are calculable. This direction allows the largest deviation from the standard model in the $S-T$ plane. It is also worth keeping in mind that it is quite possible that the estimates for the $S$ and $T$ parameters above are too large, either because our estimate for $\epsilon$ is too large or because the order- 1 factors are small. We conclude that it is very plausible that a good fit can be obtained in these models at cost of a moderate tuning.

\section{LHC signatures}

In this subsection we consider the signatures of this model at the LHC. 


\section{$5.1 h \rightarrow \gamma \gamma$ decay}

The strong sector contains charged particles with a substantial coupling to the Higgs boson, and therefore gives an important contribution to $h \rightarrow \gamma \gamma$. This is one of the important channels in which the $125 \mathrm{GeV}$ Higgs signal is present, and will be probed in the coming year of LHC running. Because the strong sector is approximately supersymmetric, the leading correction to $h \rightarrow \gamma \gamma$ is in fact calculable [13]. This is because in the low-energy effective theory below the scale $\Lambda$, the strong sector contribution can be parameterized by the effective interaction

$$
\mathcal{L}_{\text {eff }}=-\frac{1}{4 e^{2}} F^{\mu \nu} F_{\mu \nu}
$$

The low-energy effective coupling depends logarithmically on $\Lambda(H)$ due to the renormalization group. Above the scale $\Lambda(H)$, the renormalization group equation is given by the NSVZ beta function

$$
\mu \frac{d}{d \mu}\left(\frac{1}{e^{2}}\right)=-\frac{1}{16 \pi^{2}} \sum_{r} Q_{r}^{2}\left(1+\gamma_{r}\right)
$$

where

$$
\gamma_{r}=\mu \frac{d}{d \mu} \ln Z_{r}
$$

is the anomalous dimension of the chiral superfield $r$. The anomalous dimension term is negligible except for the strongly coupled fields. Assuming that the operators $\mathcal{O}_{u, d}$ in Eq. (3.1) are bilinear "meson" operators of a SUSY QCD theory, the anomalous dimension is related to the dimension of the operators by

$$
d=2-\gamma
$$

The beta function is therefore a constant above and below the threshold $\Lambda$, and the low-energy coupling depends on the scale $\Lambda(H)$ via the difference in the beta functions above and below the scale $\Lambda(H)$ :

$$
\frac{1}{e^{2}}=-\frac{3-d}{16 \pi^{2}}\left(\sum_{r} Q_{r}^{2}\right) \ln \Lambda(H)+\cdots .
$$

For example, in the minimal model of $\S 2.1$ we have

$$
\sum_{r} Q_{r}^{2}=8\left(1+4 b^{2}\right), \quad d=\frac{3}{2},
$$

where $b$ is the $B-L$ charge. 
In models where the dynamical scale is determined entirely by the Higgs VEVs, we have (see Eq. (3.5))

$$
\Lambda^{6-2 d}(H)=\Lambda_{0}^{6-2 d} \frac{H_{u}^{0} H_{d}^{0}}{v_{u} v_{d}},
$$

where $\Lambda_{0}$ is the value of the dynamical scale when the Higgs fields are replaced by their VEVs. For models where the masses in the strong sector are dominantly electroweak singlet, we have

$$
\Lambda(H)=\Lambda_{0}\left[1+\delta_{H} \frac{H_{u}^{0} H_{d}^{0}}{v_{u} v_{d}}+\cdots\right] .
$$

For the first type of model we have

$$
\mathcal{L}_{\mathrm{eff}}=\frac{e^{2}}{16 \pi^{2}} \frac{1}{4}\left(\sum_{r} Q_{r}^{2}\right) \frac{\cos (\alpha+\beta)}{\sin 2 \beta} \times \frac{h}{v} F^{\mu \nu} F_{\mu \nu},
$$

where we have canonically normalized the photon fields and used the standard definitions

$$
\begin{aligned}
& H_{u}^{0}=\frac{1}{\sqrt{2}}(v \sin \beta+h \cos \alpha+\cdots), \\
& H_{d}^{0}=\frac{1}{\sqrt{2}}(v \cos \beta-h \sin \alpha+\cdots) .
\end{aligned}
$$

The decay width is then given by (see e.g. Ref. [14])

$$
\Gamma(h \rightarrow \gamma \gamma) \propto\left|A_{\mathrm{SM}}+\frac{1}{2}\left(\sum_{r} Q_{r}^{2}\right) \frac{\cos (\alpha+\beta)}{\sin 2 \beta}\right|^{2},
$$

where $A_{\mathrm{SM}} \simeq-6.5$. For the second type of model, we obtain the same effective coupling multiplied by an additional factor of $2(3-d) \delta_{H}$.

For the minimal model with $b=0$ and $\cos (\alpha+\beta) / \sin 2 \beta \sim 1$, the width is reduced by $\frac{1}{7}$. On the other hand, with $b=1$, there is an enhancement by a factor of 4. The charge assignments in the strong sector are rather restricted if we impose the requirement that there are no states with fractional electromagnetic charges, which would give rise to cosmologically dangerous stable charged particles. For example, having only one pair of electroweak doublets in the $S U(2)$ model, the smallest $B-L$ charge we can have for the electroweak singlets is $\pm 1 / 2$, and we obtain the same value for $h \rightarrow \gamma \gamma$. However, in models with singlets, the correction to $h \rightarrow \gamma \gamma$ may be significantly reduced at the cost of a mild tuning. We conclude that generically we expect a significant deviation from the standard model rate for $h \rightarrow \gamma \gamma$, and either an increase or decrease is possible. 


\subsection{TeV Resonances}

The strong sector has hadronic resonances at the scale $\Lambda \sim 800 \mathrm{GeV}$. This scale is significantly smaller than the scale $4 \pi v \sim 2 \mathrm{TeV}$ we expect for a technicolor theory because the Higgs is weakly coupled to the strong sector. In this section, we outline some promising LHC signals of these resonances.

The strong sector is approximately supersymmetric, so the heavy resonances will appear in approximately degenerate supermultiplets. For example, the vector multiplet will also contain a scalar and a fermion. If $R$-parity is a symmetry, there will be $R$-parity even and odd resonances. The $R$-parity even resonances can be singly produced, and we will focus on their phenomenology here.

We focus on models where the strong sector has an $S U(2)_{L} \times S U(2)_{R}$ global symmetry that is weakly gauged by the standard model. To get a good precision electroweak fit, the masses induced by the Higgs VEVs must be chosen to be approximately invariant under the custodial $S U(2)_{C}$, the diagonal subgroup of $S U(2)_{L} \times S U(2)_{R}$. The full $S U(2)_{L} \times S U(2)_{R}$ is nonlinearly realized by the Higgs fields $H_{u, d}$ at the strong scale $\Lambda$. In fact, since we are considering a supersymmetric theory, we have a nonlinear realization of the complexified group [15]. Couplings to matter fields in arbitrary representations of the (complexified) $S U(2)_{C}$ are discussed in Ref. [16].

In models with unification (but no custodial symmetry), the strong fields come in complete $S U(5)$ multiplets. A good precision electroweak fit requires that the masses in the strong sector be approximately electroweak singlet, but the doublet and triplet masses need not be approximately the same. The resonances in the strong sector therefore come in complete multiplets of the standard model gauge group $S U(3)_{C} \times S U(2)_{W} \times U(1)_{Y}$.

We will focus our discussion in this section on the models with custodial symmetry, since these require less tuning. A natural candidate for the lightest resonance would be a vector supermultiplet in the $\mathbf{1}+\mathbf{3}$ representation of $S U(2)_{C}$, which would contain the singlet scalar $\sigma$, the triplet scalar $a_{0}$ and the analog of the QCD $\omega$ and $\rho$ mesons. We will not make the group theory structure explicit in the formulas below, since we are interested only in order-of-magnitude estimates.

Symmetries allow a presence of a kinetic mixing term between the Higgs fields and the singlet scalar component $\sigma$ of the resonance multiplet:

$$
\Delta \mathcal{L} \sim \frac{\Lambda}{4 \pi v} \partial^{\mu} h \partial_{\mu} \sigma \sim \epsilon \partial^{\mu} h \partial_{\mu} \sigma
$$

Electrically neutral scalar resonances can therefore be produced by gluon fusion with 
a cross section of order $\epsilon^{2} \sim 0.1$ times the gluon fusion cross section for producing a standard-model Higgs of the same mass. Through a similar mixing term for the charged Higgs boson, charged scalar resonances $a_{0}^{ \pm}$can be produced by $g b \rightarrow a_{0}^{-} t$ with a cross section of order $\epsilon^{2}$ times the cross section for a reference charged elementary Higgs of the same mass [17]. For vector resonances, there can be a mixing term between standard model vector bosons $V_{\mu}\left(Z_{\mu}\right.$, or $\left.W_{\mu}\right)$ and the vector component $\rho_{\mu}$ of the resonance of the form

$$
\Delta \mathcal{L} \sim \frac{g \Lambda^{2}}{4 \pi} V^{\mu} \rho_{\mu}
$$

where $g$ is the $V_{\mu}$ coupling constant. This allows single production of vector resonances via Drell-Yan processes with a mixing suppression of order $g / 4 \pi$. The production rate is therefore $g^{2} /(4 \pi)^{2} \sim 10^{-2}$ times that of a sequential $W^{\prime}$ or $Z^{\prime}$ of the same mass.

We now discuss decays. The largest couplings are to the Higgs fields, and longitudinal $W$ and $Z$ particles which are equal to those of the corresponding Goldstone fields by the equivalence theorem. The matrix elements of the decays of either the scalar or vector mesons to pairs of Higgs fields or Goldstones are all given by

$$
\mathcal{M} \sim \frac{\Lambda^{3}}{4 \pi v^{2}} \sim 4 \pi \epsilon^{2} \Lambda
$$

The width of the heavy resonances is therefore suppressed by $\epsilon^{4} \sim 10^{-2}$, and we expect them to be narrow. If we can neglect phase space suppression, we expect the decays to different Higgs particles and Goldstones to be comparable. A particularly interesting final states include $A A$ followed by $A \rightarrow Z h$ followed by either $h \rightarrow W W$ or $h \rightarrow \bar{b} b$. But $W_{L} W_{L}, Z_{L} Z_{L}, h h$, and $H^{+} H^{-}$are all worth further study. The decay to photons is highly suppressed because photons do not have longitudinal polarizations: $\Gamma_{\gamma \gamma} / m \sim(e / 4 \pi)^{4} \sim 10^{-4}$.

We note that this class of models is another case where a stronger Higgs sector leads to resonant production of heavy standard-model particles. The 2-Higgs doublet model is a good simplified model for many of these searches [18].

The LHC experiments have already put constraints on such resonances from resonant $W Z$ production followed by decays into 3 leptons $[19,20]$. The bound on the cross section times the branching ratio obtained there can be translated into the constraints on the $\rho$-like vector resonance. This has been done in, for example, Refs. [21, 22]. By comparing with the corresponding parameter points $\left(g_{\rho} \sim 4 \pi\right.$ and $g_{\rho \pi \pi} \sim 4 \pi \epsilon^{2}$ in Ref. [21], or $m_{\rho} \sim 800 \mathrm{GeV}$ and $a_{\rho} \sim \epsilon$ in Ref. [22]), we can see that the point is close to the current LHC bound. Therefore, the discovery of such a resonance could happen soon in these models. 


\section{Conclusions}

In this paper we have proposed models that address the naturalness problem of supersymmetric models by partial Higgs compositeness. The models consist of the MSSM or NMSSM coupled to a strong conformal sector via standard model gauge interactions and Higgs couplings of the form Eq. (3.1). The strong superconformal sector allows large Higgs couplings without Landau poles in the UV. Vacuum expectation values of the elementary Higgs fields $H_{u, d}$ and/or singlet Higgs fields break conformal symmetry in the strong sector. The strong sector has a mass scale of order $800 \mathrm{GeV}$, and generates corrections to the Higgs potential that can explain a $125 \mathrm{GeV}$ Higgs mass without any fine-tuning. The Higgs coupling also generates a Higgsino mass of order the Higgs mass, so there is no need for a $\mu$-term. On the other hand, the Higgs couplings are dimensionful relevant couplings, and the fact that they are near the $\mathrm{TeV}$ scale requires an explanation. As an example, we show that a generalization of the Giudice-Masiero mechanism can give a natural explanation.

Unlike technicolor theories, the Higgs fields are only partially composite so that we can naturally have a top-quark Yukawa coupling that is order 1. Even though electroweak symmetry is broken by the VEVs of weakly-coupled Higgs fields, there are in general large corrections to the $T$ parameter coming from the Higgs VEVs, which must be tuned away to get a good precision electroweak fit. The tuning is of order $10 \%$, significantly better than the $1 \%$ (or worse) tuning in typical supersymmetric models.

The models predict a rich phenomenology that differs in interesting ways from that of the MSSM. There are generally large corrections to the $h \rightarrow \gamma \gamma$ width, either suppression or enhancement depending on the charge assignments of the strong sector. There is also an approximately supersymmetric spectrum of hadrons at the scale $\sim 800 \mathrm{GeV}$ that decay to pairs of MSSM Higgs particles or longitudinally polarized $W$ and $Z$ s. We believe that this is a plausible framework for natural supersymmetry that is well worth further exploration.

\section{Acknowledgments}

We would like to thank A. Hebecker for discussions. MAL is supported by the US Department of Energy under grant DE-FG02-91-ER40674. RK is supported in part by the Grant-in-Aid for Scientific Research 23740165 of JSPS. YN is supported by

JSPS Fellowships for Young Scientists. YN would like to acknowledge a great debt to his former home institute, the Yukawa Institute for Theoretical Physics, Kyoto 
University where most of his work was done.

\section{Appendix: SUSY Breaking in the Conformal Sector}

In this appendix, we discuss the contribution of soft SUSY breaking terms in the strong sector to the Higgs potential. Specifically, we consider mass-squared terms proportional to generators of the flavor group and exhibit the conditions under which these lead to a contribution to the Higgs potential of the form Eqs. (3.18) and (3.42).

We consider $S U(N)$ SUSY QCD in the conformal window, i.e. $\frac{3}{2} N \leq F<3 N$, where $F$ is the number of flavors. We begin with the case $N \geq 3$, and discuss $N=2$ separately below. We add mass terms for all quarks:

$$
W=\tilde{Q}^{T} M Q
$$

In our models, $M$ is proportional to Higgs and/or singlet fields, and the scale of conformal symmetry breaking will be determined self-consistently by the VEVs of these fields.

A straightforward way to see that the symmetries of the problem do not prevent a potential of the form Eqs. (3.18) and (3.42) is to compute the 1-loop potential in the perturbative case. To be concrete, we consider a model with custodial symmetry:

$$
W=\sum_{i} y_{i} H \tilde{Q}^{i} Q^{i}
$$

The chiral superfields $Q$ and $\tilde{Q}$ are fundamental and anti-fundamental representation of the $S U(N)$ gauge group, and $i$ runs from one to $F / 2$. The one-loop effective potential is given by

$$
\begin{aligned}
V_{1-\text { loop }}=\frac{N}{(4 \pi)^{2}} \sum_{i} & {\left[\operatorname{tr}\left\{\frac{1}{2}\left(y_{i}^{2} H^{\dagger} H+m_{i}^{2}\right)^{2}\left(\ln \frac{y_{i}^{2} H^{\dagger} H+m_{i}^{2}}{\mu^{2}}-\frac{3}{2}\right)\right\}\right.} \\
+ & \operatorname{tr}\left\{\frac{1}{2}\left(y_{i}^{2} H^{\dagger} H+\tilde{m}_{i}^{2}\right)^{2}\left(\ln \frac{y_{i}^{2} H^{\dagger} H+\tilde{m}_{i}^{2}}{\mu^{2}}-\frac{3}{2}\right)\right\} \\
- & \left.\operatorname{tr}\left\{\left(y_{i}^{2} H^{\dagger} H\right)^{2}\left(\ln \frac{y_{i}^{2} H^{\dagger} H}{\mu^{2}}-\frac{3}{2}\right)\right\}\right] .
\end{aligned}
$$

Here we added soft masses $m_{i}^{2}$ and $\tilde{m}_{i}^{2}$ for $Q_{i}$ and $\tilde{Q}_{i}$, respectively. The scale $\mu$ can take an arbitrary value. The contribution at the linear order in $m_{i}^{2}$ or $\tilde{m}_{i}^{2}$ is given by

$$
V_{1-\mathrm{loop}}=\sum_{i} \operatorname{tr}\left\{\frac{y_{i}^{2} N}{(4 \pi)^{2}}\left(m_{i}^{2}+\tilde{m}_{i}^{2}\right)|H|^{2}\left(\ln \frac{y_{i}^{2}|H|^{2}}{\mu^{2}}-1\right)\right\}+O\left(m^{4}\right) \text {. }
$$


One can see that the potential is generically non-vanishing even when the soft masses are proportional to generators of the flavor group. In particular, it survives when

$$
\sum_{i}\left(m_{i}^{2}+\tilde{m}_{i}^{2}\right)=0
$$

for a generic set of $y_{i}$.

We can get a better understanding of this with a non-perturbative analysis based on symmetries, as follows. Below the scale $M$, all the quarks get massive, and the lowenergy theory is pure SUSY Yang-Mills. This theory confines, generating a dynamical scale $\Lambda$. We have

$$
d=\operatorname{dim}(\tilde{Q} Q)=3\left(1-\frac{N}{F}\right)
$$

and therefore

$$
\Lambda \propto(\operatorname{det} M)^{1 / F(3-d)}=(\operatorname{det} M)^{1 / 3 N}
$$

In a strongly coupled theory $(F \simeq 2 N)$ the proportionality constant will be order 1 .

We now consider the effects of soft SUSY breaking in the strong sector. The only soft SUSY breaking terms that are not renormalized are mass-squared terms proportional to generators of the flavor group $S U(F) \times S U(F) \times U(1)$. These can be parameterized by the $D$-term of $S U(F) \times S U(F) \times U(1)$ gauge superfields $X, \tilde{X}$

$$
X=\theta^{4} m^{2}, \quad \tilde{X}=\theta^{4} \tilde{m}^{2} .
$$

The flavor gauge fields must satisfy

$$
\operatorname{tr}(X+\tilde{X})=0
$$

to project out the anomalous $U(1)$. We write the kinetic term as

$$
\mathcal{L}_{\text {kin }}=\int d^{4} \theta\left[Q^{\dagger} e^{X} Q+\tilde{Q}^{\dagger} e^{\tilde{X}} \tilde{Q}\right]
$$

where we suppress the gauge fields. The theory is therefore invariant under the flavor gauge transformations

$$
Q \mapsto U Q, \quad \tilde{Q} \mapsto \tilde{U} \tilde{Q}
$$

and

$$
e^{-X} \mapsto U e^{-X} U^{\dagger}, \quad e^{-\tilde{X}} \mapsto \tilde{U} e^{-\tilde{X}} \tilde{U}^{\dagger}
$$


provided we let $M$ transform as

$$
M \mapsto \tilde{U}^{T-1} M U^{-1}
$$

Here $U, \tilde{U}$ are complexified flavor transformations, so $U^{\dagger} \neq U^{-1}$.

We are interested in the effective Kähler potential below the scale $\Lambda$. This must be invariant under the flavor gauge transformations above. Note that

$$
S=e^{-X} M^{\dagger} e^{-\tilde{X}^{T}} M \mapsto U S U^{-1} .
$$

We can use this to construct the invariants

$$
\operatorname{tr}\left(S^{n}\right)=\operatorname{tr}\left[\left(M^{\dagger} M\right)^{n}\right]-n \operatorname{tr}\left[X\left(M^{\dagger} M\right)^{n}+\tilde{X}^{T}\left(M M^{\dagger}\right)^{n}\right]+O\left(X^{2}\right) .
$$

The most general dynamical Kähler potential is a homogeneous function of these variables with the degree of homogeneity fixed by dimensional analysis. As long as the symmetries of the mass terms $m^{2}, \tilde{m}^{2}$ and the mass term $M$ do not force the $O(X)$ term in Eq. (A.15) to vanish, we expect a nonzero potential of the form Eqs. (3.18) and (3.42).

We also need to take into account the constraints of superconformal invariance. The mass $M$ in Eq. (A.1) is a chiral primary field with dimension $3-d$. We can write a superconformal invariant kinetic term for a chiral primary field $\Phi$ with dimension 1 as $\int d^{4} \theta \Phi^{\dagger} \Phi$. However, we cannot define $\Phi$ as a power of the matrix $M$ because this does not have a well-defined transformation under flavor gauge symmetries. The holomorphic scale $\Lambda$ is a chiral superfield of dimension 1 (see Eq. (A.7)), but it is a singlet under the flavor symmetry, and $\int d^{4} \theta \Lambda^{\dagger} \Lambda$ does not depend on the flavor gauge fields $X, \tilde{X}$. However, we can define the quantity

$$
\Delta_{b}^{a}=\frac{\partial \operatorname{det}(M)}{\partial M_{\tilde{a} a}} M_{\tilde{a} b}
$$

where $a, b, \ldots$ and $\tilde{a}, \tilde{b}, \ldots$ are $U(F) \times U(F)$ flavor indices. $\Delta \sim M^{F}$ is a chiral superfield that transforms under flavor in the adjoint representation, i.e. $\Delta \mapsto U \Delta U^{-1}$. The field

$$
\Phi=\Delta^{1 / F(3-d)} \sim \Delta^{1 / 3 N}
$$

is therefore a chiral primary field of dimension 1, and we can write the conformally invariant and flavor gauge invariant kinetic term

$$
K_{\mathrm{eff}}=\operatorname{tr}\left(\Phi^{\dagger} e^{X} \Phi e^{-X}\right)
$$


Note that in $4 \mathrm{D}$ the kinetic term for a complex scalar with gauge interactions is conformally invariant, with the gauge fields dimensionless. Note that $K_{\text {eff }}$ can be written in terms of the invariants Eq. (A.15) by writing the flavor epsilon tensors in the definition of $\Delta$ in terms of sums of Kronecker deltas. $K_{\text {eff }}$ is not the unique term invariant under all symmetries, but this is sufficient to show that the symmetries do not force the effective Kähler term to be independent of $X$.

It is straightforward to repeat the above discussion for the case of a strong $S U(2)$ gauge group. The superpotential has the form

$$
W=Q^{T} M Q
$$

where $M^{T}=-M$. The theory with $F$ favors ( $2 F$ fundamentals) has flavor group is $S U(2 F)$. Denoting the flavor gauge superfield by $X$, we can again define $S$ as in Eq. (A.14), which transforms as shown with $U$ a complexified $S U(2 F)$ transformation. We therefore have the invariants

$$
\operatorname{tr}\left(S^{n}\right)=\operatorname{tr}\left[\left(M^{\dagger} M\right)^{n}\right]-2 n \operatorname{tr}\left[X\left(M^{\dagger} M\right)^{n}\right]+O\left(X^{2}\right)
$$

It is straightforward to write $X$-dependent Kähler terms that are conformally invariant as well as flavor gauge invariant.

\section{References}

[1] For reviews, see e.g. S. P. Martin, in Kane, G.L. (ed.) Perspectives on supersymmetry [hep-ph/9709356]; M. A. Luty, hep-th/0509029; R. Kitano and Y. Nomura, Phys. Rev. D 73, 095004 (2006) [hep-ph/0602096].

[2] G. Aad et al. [ATLAS Collaboration], Phys. Lett. B 710, 49 (2012) [arXiv:1202.1408 [hep-ex]]; S. Chatrchyan et al. [CMS Collaboration], arXiv:1202.1488 [hep-ex].

[3] A. Azatov, J. Galloway and M. A. Luty, Phys. Rev. Lett. 108, 041802 (2012) [arXiv:1106.3346 [hep-ph]]; A. Azatov, J. Galloway and M. A. Luty, Phys. Rev. D 85, 015018 (2012) [arXiv:1106.4815 [hep-ph]].

[4] T. Gherghetta and A. Pomarol, JHEP 1112, 069 (2011) [arXiv:1107.4697 [hepph]].

[5] J. J. Heckman, P. Kumar, C. Vafa and B. Wecht, JHEP 1201, 156 (2012) [arXiv:1108.3849 [hep-ph]]. 
[6] H. Fukushima, R. Kitano, M. Yamaguchi, JHEP 1101, 111 (2011) [arXiv:1012.5394 [hep-ph]].

[7] J. L. Evans, M. Ibe and T. T. Yanagida, arXiv:1204.6085 [hep-ph].

[8] N. Arkani-Hamed, R. Rattazzi, Phys. Lett. B454, 290-296 (1999) [hepth/9804068]; A. Karch, T. Kobayashi, J. Kubo, G. Zoupanos, Phys. Lett. B441, 235-242 (1998) [hep-th/9808178].

[9] M. A. Luty and R. Rattazzi, JHEP 9911, 001 (1999) [hep-th/9908085].

[10] M. A. Luty, Phys. Rev. D57, 1531-1538 (1998), [hep-ph/9706235].

[11] M. E. Peskin, T. Takeuchi, Phys. Rev. Lett. 65, 964-967 (1990); M. E. Peskin, T. Takeuchi, Phys. Rev. D46, 381-409 (1992).

[12] L. Lavoura, J. P. Silva, Phys. Rev. D47, 2046-2057 (1993).

[13] J. J. Heckman, P. Kumar and B. Wecht, arXiv:1204.3640 [hep-ph].

[14] M. Spira, A. Djouadi, D. Graudenz and P. M. Zerwas, Nucl. Phys. B 453, 17 (1995) [hep-ph/9504378].

[15] B. Zumino, Phys. Lett. B 87, 203 (1979).

[16] M. Bando, T. Kuramoto, T. Maskawa and S. Uehara, Phys. Lett. B 138, 94 (1984); Prog. Theor. Phys. 72, 313 (1984); Prog. Theor. Phys. 72, 1207 (1984).

[17] J. A. Evans and M. A. Luty, Phys. Rev. Lett. 103, 101801 (2009) [arXiv:0904.2182 [hep-ph]].

[18] S. Chang, J. A. Evans and M. A. Luty, Phys. Rev. D 84, 095030 (2011) [arXiv:1107.2398 [hep-ph]]. See also the contribution in D. Alves et al. [LHC New Physics Working Group Collaboration], arXiv:1105.2838 [hep-ph].

[19] G. Aad et al. [ATLAS Collaboration], arXiv:1204.1648 [hep-ex].

[20] S. Chatrchyan et al. [CMS Collaboration], arXiv:1206.0433 [hep-ex].

[21] A. Falkowski, C. Grojean, A. Kaminska, S. Pokorski and A. Weiler, JHEP 1111, 028 (2011) [arXiv:1108.1183 [hep-ph]].

[22] B. Bellazzini, C. Csaki, J. Hubisz, J. Serra and J. Terning, arXiv:1205.4032 [hep$\mathrm{ph}$. 